\title{
Article \\ Genome-Wide Identification of the Thaumatin-like Protein Family Genes in Gossypium barbadense and Analysis of Their Responses to Verticillium dahliae Infection
}

\author{
Yilin Zhang ${ }^{1, \dagger}$, Wei Chen ${ }^{2,+}$, Xiaohui Sang ${ }^{2}$, Ting Wang ${ }^{1}$, Haiyan Gong ${ }^{2}$, Yunlei Zhao ${ }^{1,2, *}$, Pei Zhao ${ }^{1,2, *}$ \\ and Hongmei Wang 1,2,* \\ 1 Zhengzhou Research Base, State Key Laboratory of Cotton Biology, School of Agricultural Sciences, \\ Zhengzhou University, Zhengzhou 450001, China; zhangyilin19971112@163.com (Y.Z.); \\ tingwang2021@126.com (T.W.) \\ 2 State Key Laboratory of Cotton Biology, Institute of Cotton Research of Chinese Academy of Agricultural \\ Sciences, Anyang 455000, China; chenwei01@caas.cn (W.C.); sangxiaohui@caas.cn (X.S.); \\ gonghaiyan@caas.cn (H.G.) \\ * Correspondence: zhaoyunlei@caas.cn (Y.Z.); zhaopei@caas.cn (P.Z.); wanghongmei@caas.cn (H.W.) \\ + These two authors contributed equally to this work.
}

check for

updates

Citation: Zhang, Y.; Chen, W.; Sang, X.; Wang, T.; Gong, H.; Zhao, Y.; Zhao, P.; Wang, H. Genome-Wide Identification of the Thaumatin-like Protein Family Genes in Gossypium barbadense and Analysis of Their Responses to Verticillium dahliae Infection. Plants 2021, 10, 2647. https://doi.org/10.3390/ plants10122647

Academic Editor: Qian-Hao Zhu

Received: 14 October 2021

Accepted: 5 November 2021

Published: 2 December 2021

Publisher's Note: MDPI stays neutral with regard to jurisdictional claims in published maps and institutional affiliations.

Copyright: (c) 2021 by the authors. Licensee MDPI, Basel, Switzerland. This article is an open access article distributed under the terms and conditions of the Creative Commons Attribution (CC BY) license (https:/ / creativecommons.org/licenses/by/ $4.0 /)$.

\begin{abstract}
Background: Plants respond to pathogen challenge by activating a defense system involving pathogenesis-related (PR) proteins. The PR-5 family includes thaumatin, thaumatin-like proteins (TLPs), and other related proteins. TLPs play an important role in response to biotic and abiotic stresses. Many TLP-encoding genes have been identified and functionally characterized in the model plant species. (2) Results: We identified a total of 90 TLP genes in the G. barbadense genome. They were phylogenetically classified into 10 subfamilies and distributed across 19 chromosomes and nine scaffolds. The genes were characterized by examining their exon-intron structures, promoter ciselements, conserved domains, synteny and collinearity, gene family evolution, and gene duplications. Several TLP genes were predicted to be targets of miRNAs. Investigation of expression changes of 21 GbTLPs in a G. barbadense cultivar (Hai7124) resistance to Verticillium dahliae revealed 13 GbTLPs being upregulated in response to $V$. dahliae infection, suggesting a potential role of these GbTLP genes in disease response. (3) Conclusions: The results of this study allow insight into the GbTLP gene family, identify GbTLP genes responsive to $V$. dahliae infection, and provide candidate genes for future studies of their roles in disease resistance.
\end{abstract}

Keywords: GbTLP; Verticillium wilt; expression patterns

\section{Introduction}

Plants are usually exposed to a wide range of pathogens throughout their life cycle, which often triggers complex defense mechanisms, such as upregulation of pathogenesisrelated (PR) genes in response to pathogen attacks. Thaumatin-like proteins (TLPs) are a member of PR protein family 5 (PR-5 protein) and are identified by their highly conserved thaumatin domain. Generally, the majority of TLPs contain the following conserved family signature sequence: $G-x-\{G F\}-x-C-x-T-\{G A\}-D-C-x(1,2)-\{G Q\}-x(2,3)-C[1,2]$. They also contain 16 conserved Cys residues and an REDDD (glutamic acid, arginine and three aspartic acid residues) structure. The eight disulfide bonds in the structure contribute to maintaining the stability of the protein structure, allowing the protein to resist acid, alkali, and protease degradation and heat-induced denaturation [3]. Typical TLPs have a three-dimensional structure which possesses three conserved domains, namely, I, II, and III, and forms a V-shaped acidic cleft on the surface, ensuring the catalytic function of TLPs $[3,4]$.

The TLPs have been shown to exhibit antifungal functions, covering inhibition of fungal enzymes ( $\beta$-glucanase, xylanase, $\alpha$-amylase, and trypsin), as well as the ability to 
lyse fungal cell membranes and spores, and to decrease the viability of germinated spores and induce programmed cell death in fungi $[5,6]$.

TLPs are ubiquitous proteins present in fungi, plants, and animals $[7,8]$. To date, genome-wide analysis has been used to identify three TLP genes in the basidiomycete Moniliophthora perniciosa, two TLP genes in Cryptococcus neoformans, five TLP genes in the flour beetle Tribolium castaneum, six TLP genes in the pea aphid Acyrthosiphon pisum, and six TLP genes in the nematode Caenorhabditis elegans [4]. In contrast, plants have a higher number of TLP genes, with 28 in Arabidopsis thaliana [2], 31 in Oryza sativa [2], 33 in Vitis vinifera [9], 28 in Cucumis melo [10], and 55 in Populus trichocarpa [1], as well as 50, 48, 91, and 90 in Gossypium raimondii, G. arboreum, G. barbadense, and G. hirsutum, respectively [11].

Transgenic plants overexpressing TLP have been shown to enhance resistance against different pathogenic fungi $[9,12]$. In vitro antifungal activity of leaf extracts from transgenic tobacco and grape expressing exogenous TLPs has been reported to inhibit mycelial growth of Pythium aphanidermatum, Rhizoctonia solani, Uncinula necator, Botrytis cinerea, and Elsinoë ampelina [9,13-15]. To date, endogenous TLPs extracted from banana, peach, and kiwifruit have been proven to be potent antifungal proteins [16,17]. TLPs involved in plant defense responses have been well studied in O. sativa and other model systems, with overexpression of an OsTLP gene in cassava and banana leading to significantly enhanced resistance against Colletotrichum gloeosporioides f. sp. manihotis and Fusarium oxysporum $[18,19]$. Transgenic tobacco overexpressing AdTLP gene, from the wild peanut Arachis diogoi, showed enhanced resistance to the fungal pathogen, Rhizoctonia solani [20]. Furthermore, transgenic potato plants overexpressing CSTLP from tea (Camellia sinensis) exhibited increased resistance against two fungal pathogens [21]. Expression of endogenous TLP genes in melon was markedly upregulated in response to Podosphaera xanthii infection, and the TLPs were also expressed differentially in different tissues and organs [10]. In addition, TLPs in a range of fruits and pollen of apple, cherry, olive, banana, tomato, wheat, and cypress have allergenic activity [22]. Transgenic tobacco plants with constitutively higher expression of the GbTLP1 showed enhanced resistance against Verticillium dahliae, Fusarium oxysporum, and some abiotic stresses including salinity and drought [23]. The GhTLP19-silenced cotton exhibited increased sensitivity to Verticillium dahliae via increasing the malondialdehyde content and decreasing the catalase content [11].

Globally, cotton is one of the most widely grown commercial crops. It offers not only fibers but also cottonseed oil to the world economy. Nevertheless, cotton productivity has been constrained by a number of biotic and abiotic factors, which cause enormous losses worldwide. A particular yield-limiting factor is the disease Verticillium wilt (VW) caused by Verticillium dahliae, which is the most destructive fungal disease affecting cotton production in the world [24-26]. V. dahliae is a soil-borne fungus, which can survive in soil for many years and has a wide host range of over 400 plant species [27]. V. dahliae mainly damages the roots of cotton, causing leaf yellowing, the browning of vascular bundles, the dehiscence of flower buds and bolls, and even the death of the whole plant [28]. At present, the main methods for controlling VW of cotton include resistance breeding and cultural, fungicidal, and biological control. However, in terms of actual production, it is difficult to implement crop rotation due to factors such as land competition between grain and cotton, problems with finding suitable nonhost crops, and its time-consuming and laborious nature. In terms of chemical control, traditional fungicides, which pose a threat to the environment and human health, have been gradually eliminated, while new fungicidal chemicals have an inadequate control spectrum, in addition to retaining potential negative impacts on the environment. Biological control has some limitations, and its control effect is slow and easily limited by climatic and local environmental factors, while the control effect is variable and unstable. Therefore, increasing host resistance is the main focus for controlling VW. 


\section{Results}

\subsection{Identification of TLP Genes in the Sea Island Cotton Genome}

Using the hidden Markov model (HMM) profile of the thaumatin domain (PF00314) and the TLP sequences from Arabidopsis $(n=28)$ and O. sativa (31) as queries, a total of 90 predicted TLP gene sequences were identified from G. barbadense. The 90 TLP genes were then sorted according to their chromosomal distribution and relative linear location and named GbTLP1 to GbTLP90, with detailed information listed in Table S1. The 90 TLP genes in the sea island cotton genome encoded proteins with different numbers of amino acids. The predicted TLP genes encoded proteins ranging in size between 175 (TLP64) and 788 (TLP66) amino acids, with predicted molecular weights varying between 18.65 and $86.97 \mathrm{kDa}$. The isoelectric point (pI) ranged from 4.10 to 9.22 (Table S1).

\subsection{Chromosomal Distribution of GbTLPs and Gene Collinearity Analysis}

Using the genome sequence of sea island cotton as reference, the 90 TLP genes identified were mapped onto chromosomes or scaffolds by MapChart. The 90 GbTLP genes were distributed across 19 chromosomes and nine scaffolds, with 41 TLP genes distributed on $10 \mathrm{Dt}$ chromosomes and 35 TLP genes allocated to nine At chromosomes, with the remaining $14 T L P$ genes being located on nine scaffolds (Figure 1). The chromosome with the most genes in the At subgenome is A11, which has seven TLP genes, compared with nine TLP genes on chromosome D11 from the Dt subgenome. The sea island cotton genome has five chromosomes (chromosomes A02, A03, D03, and D12, and scaffold_0365) each carrying four TLP genes, three chromosomes (chromosomes A01, A05, and D01) each carrying three TLP genes, and four chromosomes (chromosomes A04, A12, D02, and D05) each possessing five TLP genes. Moreover, there are six, seven, and nine TLP genes respectively distributed on chromosomes D04, A11, and D11, while the other seven chromosomes (chromosomes D06, D10, scaffold_0862, scaffold_0973, scaffold_1028, scaffold_1160, scaffold_1713, and scaffold_1950) each contain only one TLP gene (Figure 1).

Meanwhile, the mechanism of expansion of the TLP gene family was studied by performing a gene duplication event analysis, including tandem duplication and segmental duplication in the cotton species. There were 135 pairs of duplicated GbTLPs genes identified in G. barbadense. Among them, 13 TLP genes (GbTLP6, GbTLP7, GbTLP73, GbTLP72, GbTLP59, GbTLP60, GbTLP78, GbTLP79, GbTLP80, GbTLP85, GbTLP86, GbTLP87, and GbTLP88) were clustered in six tandem repeat event regions (namely, chromosomes A02, A12, and D06 and scaffold_0365, scaffold_1296, and scaffold_1326) in the sea island cotton genome, which agreed with the findings of the chromosomal location studies that some genes congregate at certain positions on particular chromosomes (Figure 2). There were two pairs of tandem duplicated genes (GbTLP78 and GbTLP79, GbTLP79 and GbTLP80) within the scaffold region of scaffold_0365, suggesting that GbTLP78 and GbTLP80 may have arisen from the same duplication event as GbTLP79. In addition, 120 pairs of segmental duplications were found within GbTLP in sea island cotton (Figure 2). Notably, all of the segmental duplication gene pairs identified were distributed on different chromosomes in the cotton genome. However, the segmental duplication gene pairs on chromosomes were unevenly distributed; there were 26 pairs of genes duplicated between the Dt subgroups, 23 pairs of genes duplicated between the At subgroups, and only seven pairs of segmental duplicates duplicated between chromosomes and scaffolds. Interestingly, most of the segmental duplication events occurred in 64 pairs of GbTLP genes between the At subgroups and Dt subgroups (Figure 2). In addition, of all the segmental duplication events, most occurred between A11 and D11, involving eight pairs of genes. 


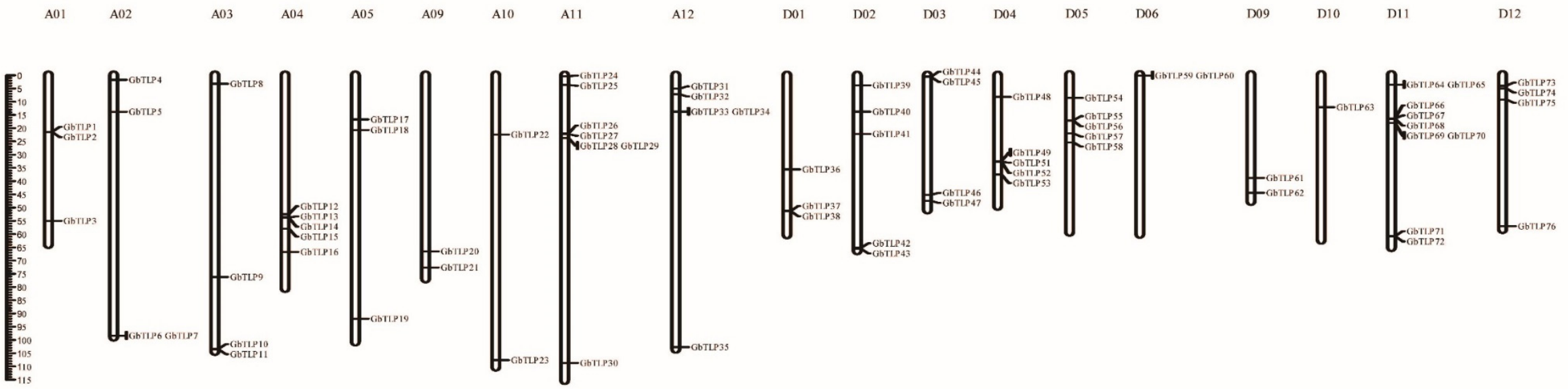

scaffold_0365.UKA scaffold_0862.UKA scaffold_0973.UKA scaffiol__1028.UKA scaffold_1160.A03 scaffold_1296.A05 scaffold_1326.A06 scaffold_1713.D02 scaffold_1950.D1
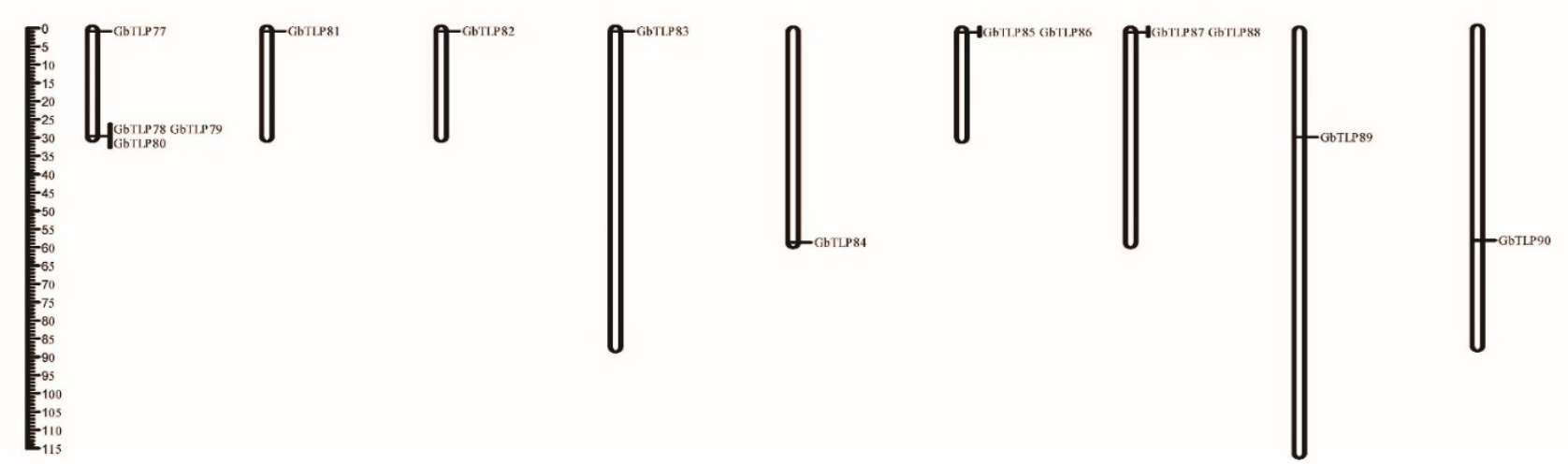

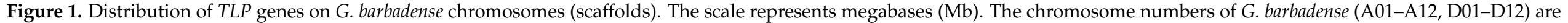
indicated above each vertical bar. 


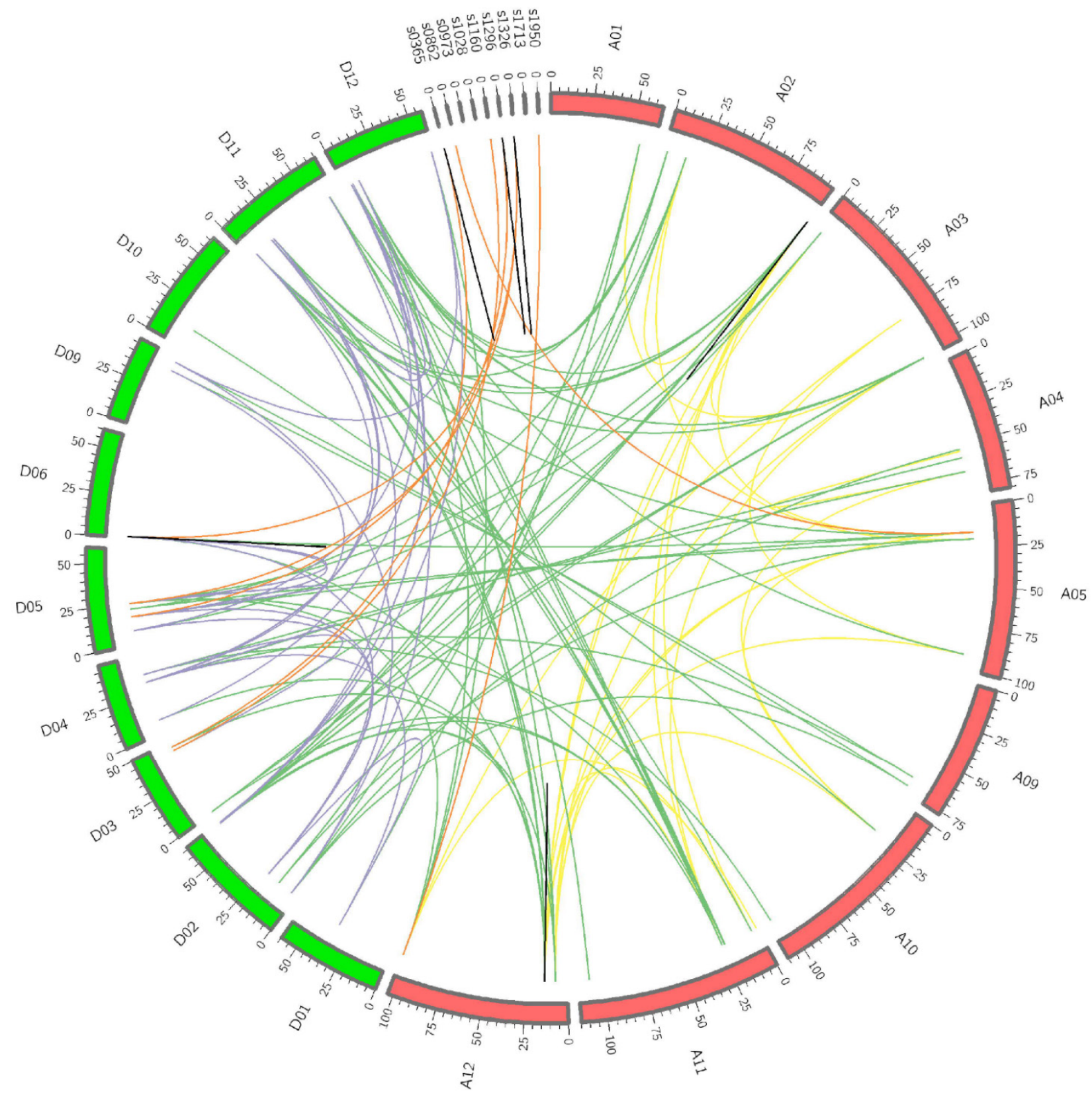

Figure 2. Distribution of segmentally duplicated and tandem duplicated TLP genes in the sea island genome. The segmental duplication gene pairs were linked by the lines between chromosomes. The putative TLP genes between At and Dt, At and At, Dt and Dt, and chromosomes and scaffolds are connected by green, yellow, purple, and red lines, respectively, while the tandem duplicates are denoted by black lines.

\subsection{Phylogenetic and Evolutionary Analysis}

In order to further analyze the evolutionary relationship of TLP genes among different species, $118 T L P$ genes were identified from $A$. thaliana $(n=28)$ and G. barbadense $(n=90)$, and corresponding phylogenetic trees were constructed (Figure 3). These TLP genes could be clearly divided into 10 paraphyletic groups according to their phylogenetic relationship, named groups 1-10 (Figure 3). The results were consistent with previous studies in other plant species, such as A. thaliana, Cucumis melo, and P. trichocarpa $[1,7,10]$. Each of these subgroups contained at least one AtTLP gene, indicating the close relationship between GbTLPs and those of other plants. The largest of these groups was group 10, which 
contained 30 GbTLP genes and 10 AtTLP genes, whereas the smallest was group 2, which contained three GbTLPs and one AtTLP (Figure 3). Multiple studies have shown that the gene members in group 5 from other species can respond to pathogenic or environmental stress, and TLPs with antifungal activity tend to have $\beta-1,3$-glucanase activity $[20,29]$. The glucanase activity enables the TLPs to bind to and degrade $\beta-1,3$-glucan, the main component of fungal cell walls [30]. This result suggests that the members of this group may play a significant role in plant response to fungal pathogen stress, a finding which is worthy of further research. Analysis of the phylogenetic tree of the TLP gene family in group 5 identified 12 GbTLP genes, namely, GbTLP8, GbTLP13, GbTLP30, GbTLP47, GbTLP49, GbTLP71, GbTLP72, GbTLP77, GbTLP78, GbTLP79, GbTLP80, and GbTLP83, which are potential candidate genes involved in disease resistance.

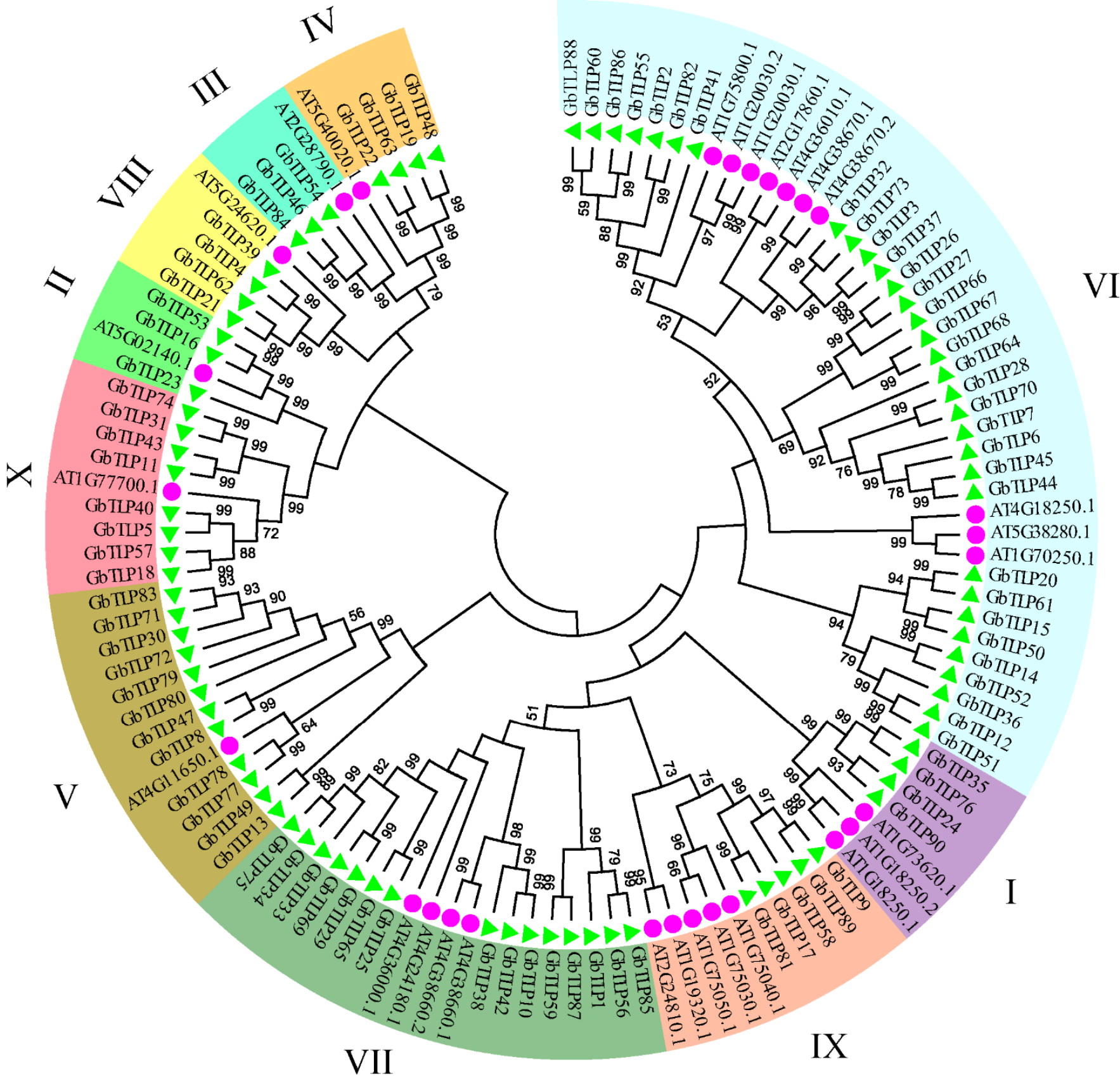

Figure 3. Phylogenetic analysis of TLPs. AT represents A. thaliana (purple circle); Gb represents G. barbadense (green triangle). The phylogenetic tree was generated using the amino-acid sequences of selected TLPS via NJ methods. All sea island cotton $T L P \mathrm{~s}$, together with their A. thaliana counterparts, were classified into 10 groups. 
Natural selection analysis is widely used to estimate how selection pressure and evolutionary forces affect duplicated genes and their corresponding proteins [31]. In order to investigate the evolutionary origin of the GbTLP genes and to further understand the divergence of the sea island cotton TLP gene family after polyploidization, estimates of the nonsynonymous (Ka) and synonymous (Ks) nucleotide substitution rates during evolution were calculated, using TBtools, allowing the calculation and analysis of the ratio $(\mathrm{Ka} / \mathrm{Ks})$, and using the $\mathrm{Ka} / \mathrm{Ks}$ ratio to estimate the balance between neutral selection $(\mathrm{Ka} / \mathrm{Ks}=1)$, purifying (negative) selection $(\mathrm{Ka} / \mathrm{Ks}<1)$, and positive selection $(\mathrm{Ka} / \mathrm{Ks}>1)$. The $\mathrm{Ka} / \mathrm{Ks}$ ratio is a measure used to investigate the mechanisms of evolution of a duplicated gene following its divergence from its ancestral gene [32]. According to the results, 132 of the $135 \mathrm{GbTLP}$ paralogous pairs had $\mathrm{Ka} / \mathrm{Ks}$ ratios $<1$, suggesting that the GbTLP gene family of sea island cotton was mainly affected by high levels of purifying selection. Meanwhile, the remaining three pairs of duplicated genes had a Ka/Ks ratio > 1 (TLP7 and TLP6, TLP18 and TLP57, TLP30 and TLP72), indicating that they evolved under positive selection. In conclusion, the evolution of the TLP gene family in sea island cotton was mainly subject to purifying selection, with only a few genes attracting positive selection. At the protein level, the result revealed that the GbTLPs evolved slowly, with conserved structure.

\subsection{Motif Composition and Gene Structure Analyses}

Exon-intron structural diversity often plays a key role in the evolution of gene families and can provide additional evidence to support phylogenetic analysis [33]. To gain further insight into the diversification of the TLP genes in sea island cotton, the exon-intron organization and conserved motifs were further analyzed (Figure 4). The structural analysis of GbTLP genes revealed that the numbers of exons in each gene varied between one and 11. As is well known, different combinations of exons and introns can lead to diverse gene functions. In Figure $4 a, c$, similar gene structures are shown in the same group, with gene clustering. Compared with the greater variation in the size of the introns, the approximate sizes of the exons were relatively highly conserved among the sea island cotton TLP genes (Figure 4c). The protein motifs are highly conserved amino-acid residues that are considered to possibly have functional and/or structural roles in active proteins. In the current study, motif distributions of the 90 thaumatin-like proteins in sea island cotton were analyzed using the MEME program, and 10 conserved motifs, designated motifs 1-10, were identified (Figure 4b). The similar motif arrangements among all 90 GbTLPs indicated that the protein structure of the TLPs was conserved in sea island cotton. This is consistent with previous research on the TLP gene family, which has been found to be highly conserved, although the functions of most of these conserved motifs remain to be elucidated. Of these, motif 5, motif 2, motif 1 , and motif 9 were present in all GbTLPs, except for GbTLP33, GbTLP78, GbTLP64, and GbTLP19, respectively. Overall, motifs 1, 2, 3, $4,5,8$, and 9 were detected in all TLP members of sea island cotton. In most cases, splice variants of GbTLPs showed similar protein sequences with a loss of the motif 2. Despite small differences in motif types among groups, members within the same group tended to exhibit similar motif patterns, such as GbTLP82, GbTLP88, GbTLP60, and GbTLP86, indicating that the functions between them might be extremely similar. It was confirmed that the diversity of TLP gene evolution was affected by gene structure and motif patterns. 


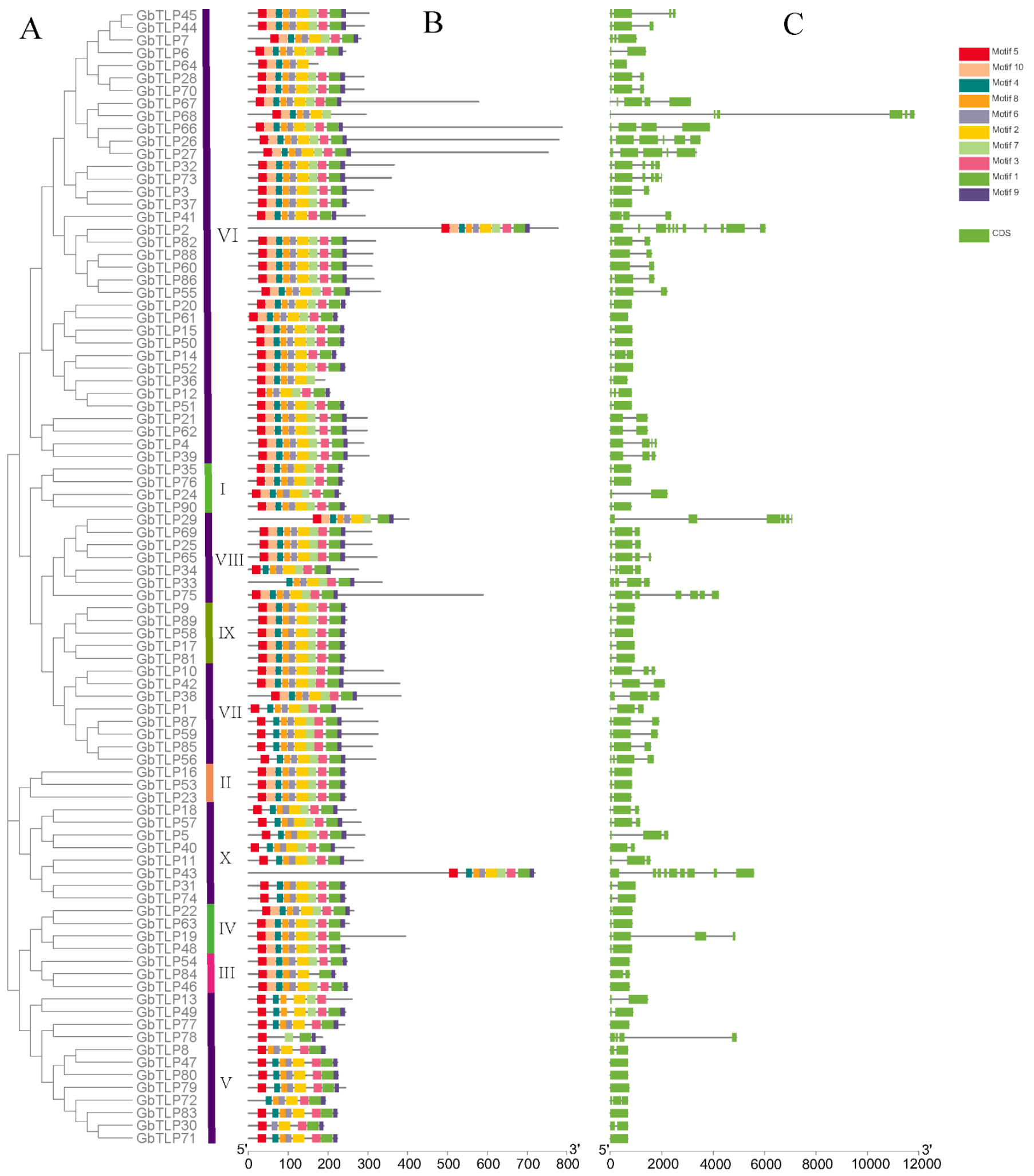

Figure 4. Cluster analysis, gene structure, and domain analysis of sea island cotton TLP gene family. (A) Phylogenetic tree of G. barbadense TLP constructed with MEGA 7.0 by the NJ method. Bootstrap values from 1000 replicates are indicated at each branch. (B) Domain compositions of sea island cotton TLP. (C) Exon-intron structures of GbTLP genes. 


\section{5. cis-Element Analysis of GbTLP Promoters}

The cis-acting regulatory element is a specific promoter motif to which an appropriate transcription factor binds in order to regulate gene transcription in plants [34]. We regarded the $2.5 \mathrm{~kb}$ genomic sequences upstream of the transcription start site (TSS) of each sea island cotton TLP gene as putative promoter regions and used the PlantCARE (http:/ / bioinformatics.psb.ugent.be/webtools/plantcare/) (22 October 2019) tool to identify the presence of cis-elements. All 90 GbTLP promoters possessed the typical core cis-acting elements in promoter regions, including TATA and CAAT boxes. A total of 2753 cis-acting elements were observed in the promoter regions of GbTLP genes (Figure 5). There were 15 types of cis-acting elements, the functions of which included stress response, hormone regulation, cellular development, MYB-binding sites, and metabolic regulation. The results revealed that five types of stress response, namely, anaerobiosis (ARE), drought (MBS), cold stress (LTR), wound stress (WUN-motif), and defense stress (TC-rich repeats), were identified in the GbTLP promoter regions. Furthermore, 11 types of hormone regulation elements, namely, ABRE, AuxRR-core, TGA-box, TGA-element, GARE-motif, TATC-box, P-box, CGTAC-motif, TGACG-motif, SARE-element, and TCAelement, which were associated with abscisic acid (ABA), gibberellin (GA), auxin (IAA), methyl jasmonate (MeJA), and salicylic acid (SA) responses, were found in the GbTLP promoters. All of them exist in GbTLP family members and respond to at least one hormone. Another element, involved in the regulation of cellular development, involves only one of the cis-elements, HD-Zip 1, which was associated with cell differentiation, and which is present in five GbTLP genes (GbTLP27, GbTLP34, GbTLP66, GbTLP75, and GbTLP84). The cis-elements of the $\mathrm{O}_{2}$-site and circadian were associated with zein metabolism regulation and circadian regulation, respectively, and were present in 27 and 12 GbTLP genes, respectively. In addition, many light-responsive elements were present in GbTLP promoters. The results showed that different elements in the promoter region of the GbTLP gene family may play important roles in regulating plant growth, abiotic stress tolerance, and different hormone responses (Figure 5).

\subsection{Identification of GbTLPs Targeted by miRNAs}

MicroRNAs (miRNAs) are endogenous noncoding small RNAs, 19-25 nucleotides long, which play important roles in the regulation of plant stress response. They were first discovered in nematodes and are now known to be ubiquitous in eukaryotes [35,36]. miRNAs bind to their target mRNA and initiate the mRNA degradation machinery, leading to mRNA degradation or, in some cases, translational repression. miRNAs play essential roles in metabolism, tissue growth, organ development and differentiation, and apoptosis in plants [37-39]. A total of 64 miRNAs associated with VW response were identified from the reported literature. To predict miRNA-mediated post-transcriptional regulation of GbTLPs, we searched GbTLP coding sequences for target sites of miRNAs, using the psRNATarget server with stricter parameters than the defaults, and predicted 12 GbTLPs as targets of five miRNAs (Figure 6). The results showed that GbTLP35, GbTLP59, and GbTLP87 were each targeted by miR855, while GbTLP26, GbTLP27, and GbTLP67 were each targeted by miR158a; furthermore, miR7488 targeted GbTLP32 and GbTLP73, whereas GbTLP23 and GbTLP75 were each targeted by miR529b, and miR2595 targeted GbTLP29 and GbTLP66. All these target sites are located in the thaumatin domain (Figure 6). Our results revealed that the miRNA-mediated post-transcriptional regulation of TLP expression might be important for response to VW infection in cotton. 


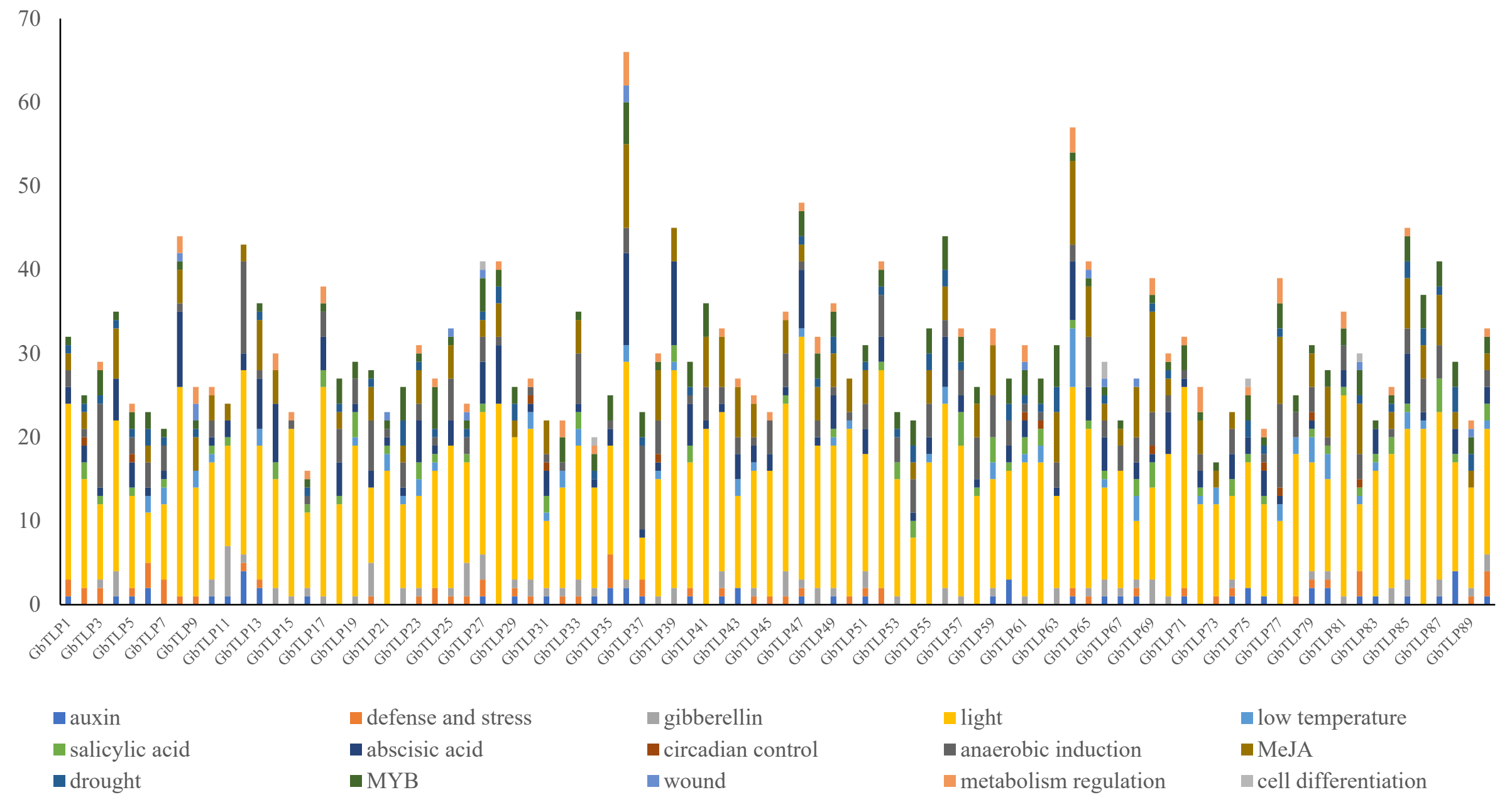

Figure 5. cis-Element analysis of putative GbTLP promoters. Different cis-elements with the same or similar functions are shown in the same color. 
GbTLP23

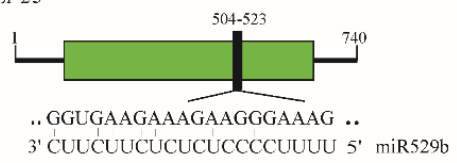

GbTLP29

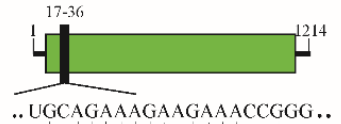

.. UGCAGAAAGAAGAAACCGGG..
3' UCUUCUUUUCUUCUUUUUACCU 5' miR2595

GbTIP59

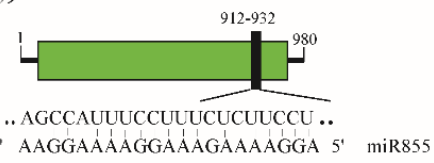

GbTLP73

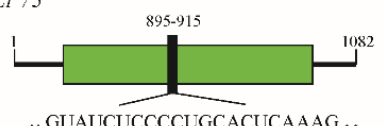

3'AAAÁCAGGGG'ÁCAUGAGUUUU' miR7488
(G)TLP26

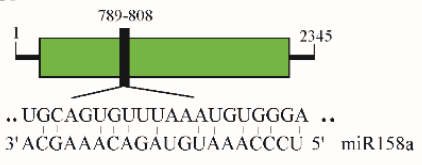

GbTLP32

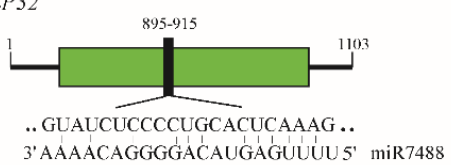

GbILI'66

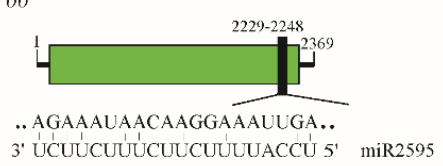

GbTLP75

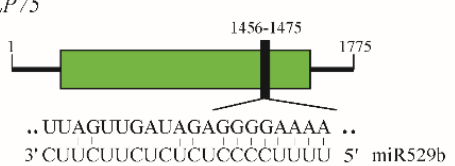

(ibTLP27

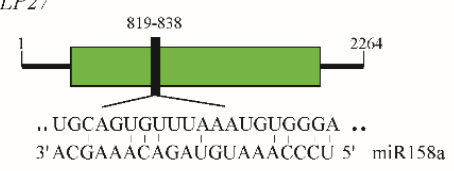

GbTLP35

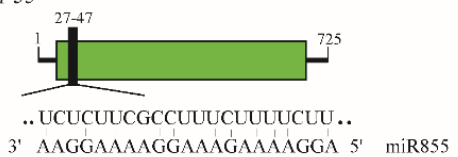

GbTIP67

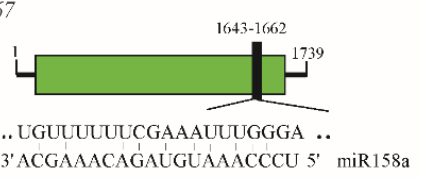

(GBTLP87

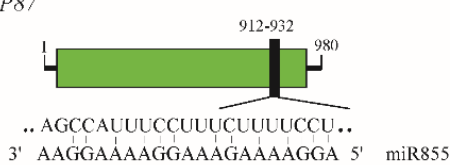

Figure 6. The prediction of targeting regulatory relations between GbTLP and miRNAs. Black lines represented ORFs of GbTLPs. The thaumatin domains are represented by boxes filled with green. miRNA complementary sites (black filling) with the nucleotide positions of GbTLP cDNAs are highlighted. The RNA sequence of each complementary site from $5^{\prime}$ to $3^{\prime}$ and the predicted miRNA sequence from $3^{\prime}$ to $5^{\prime}$ are shown in the expanded regions.

\subsection{Differential Expression of GbTLP Genes in Response to V. dahliae Infection}

To test whether the expression of GbTLP genes was influenced by challenge of $V$. dahliae, 21 GbTLP members, whose homologs in other plant species were potentially associated with disease resistance according to the previous studies, were analyzed for their responses to $V$. dahliae infection in cotton. The cultivar used in the expression experiments was G. barbadense cv. Hai 7124, which is a VW-resistant cultivar. qPCR experiments were performed to analyze the expression patterns of individual GbTLP genes in response to challenge by $V$. dahliae. Overall, expression levels of some GbTLP genes were significantly up- or downregulated following inoculation with $V$. dahliae compared to the control $(0 \mathrm{~h})$ (Figure 7). Expression of 13 of these genes was upregulated at $6 \mathrm{~h}$ to $24 \mathrm{~h}$ after inoculation with $V$. dahliae, whereas expression of three genes (GbTLP13, GbTLP49, and GbTLP72) was downregulated, with the expression of GbTLP13, for example, decreasing after $V$. dahlia infection, reaching the lowest level at 6-12 h, suggesting that these downregulated genes may play crucial biological roles in the VW response in cotton. The remaining genes showed a relatively stable expression at all times in infected and uninfected plants, indicating that they are probably not associated with resistance to $\mathrm{VW}$ in cotton. These results suggest that a subset of GbTLP genes may play a role in VW resistance in G. barbadense. 
GbTLP5

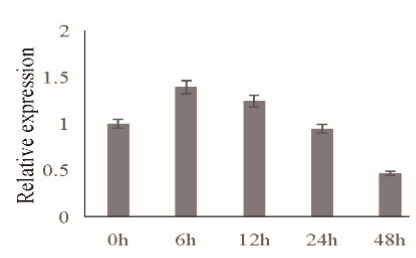

GbTLP17

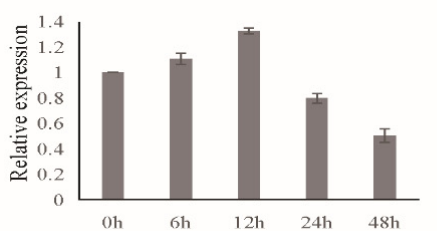

GbTLP35

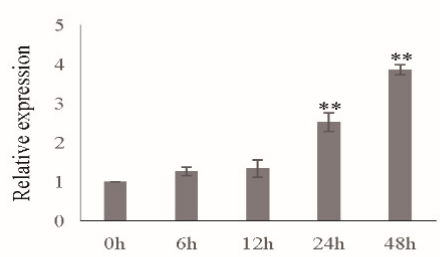

GbTLP47

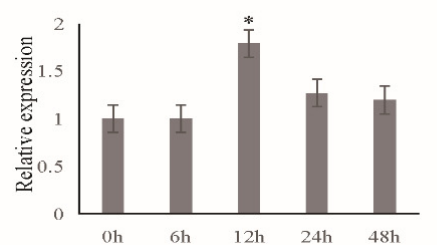

GbTLP71

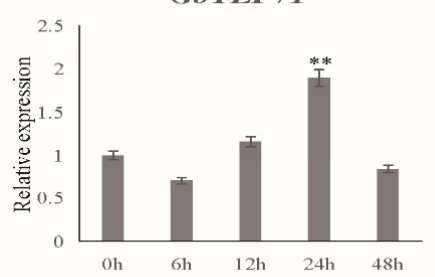

GbTLP77

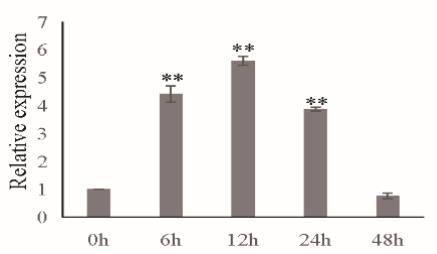

GbTLP80

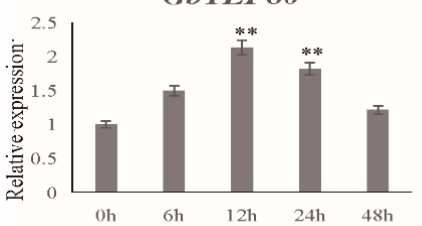

GbTLP8

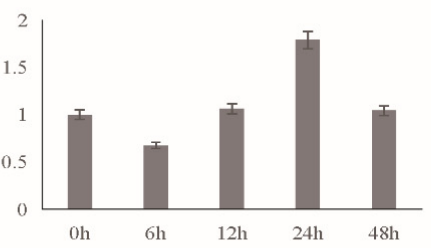

GbTLP30

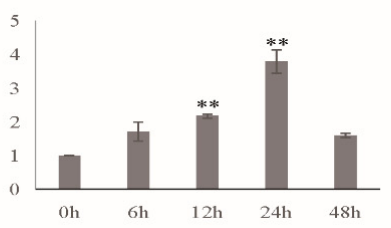

GbTLP39

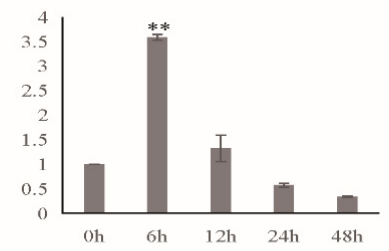

GbTLP49

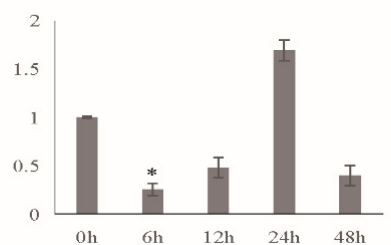

GbTLP72

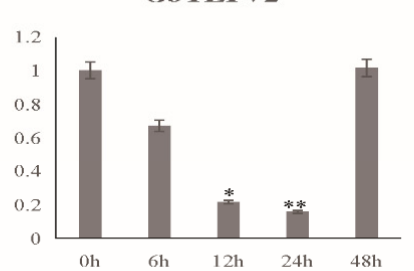

GbTLP78

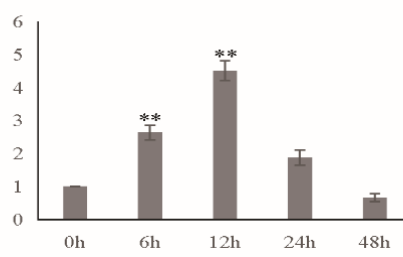

GbTLP83

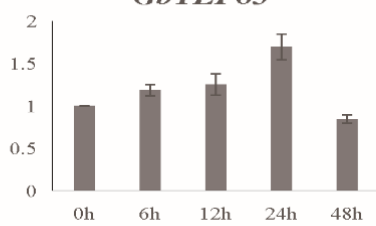

GbTLP13

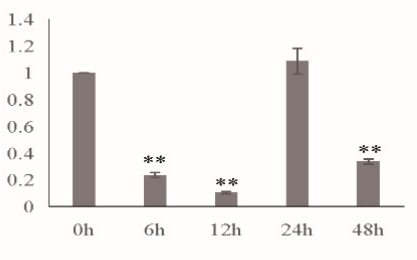

GbTLP31

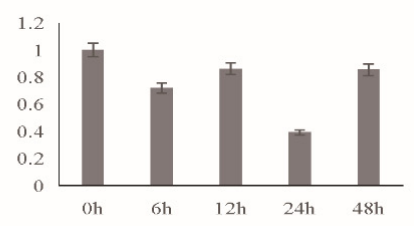

GhTLP4O

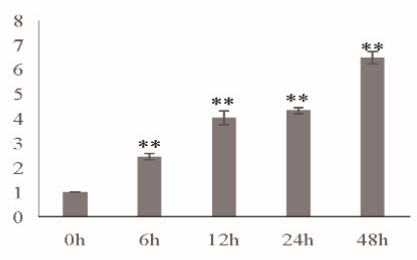

GbTLP70

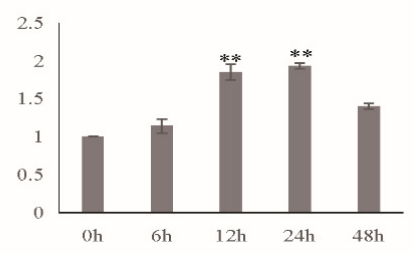

GbTLP76

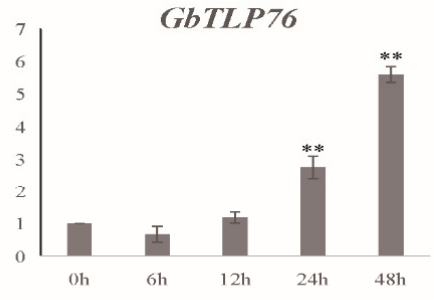

GbTLP79

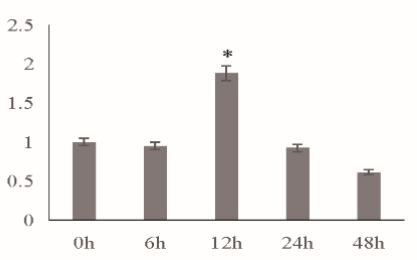

GbTLP9O

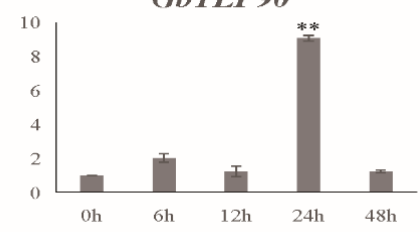

Figure 7. Gene expression of identified GbTLP after VW infection. Dark bars represented the relative expression of TLP in Hai 7124. Relative expression was calculated using the comparative threshold $2^{-\Delta \Delta C T}$ method, and values represent averages of three independent biological replicates of three plants each. Error bars were calculated on the basis of three biological replicates using standard deviation; ${ }^{*} p<0.05$ and ${ }^{* *} p<0.01$ by Student 's $t$-test. 


\section{Discussion}

Thaumatin-like proteins (TLPs) are a large family of plant PR-5 proteins that play a critical role in disease resistance [1]. Genome-wide analyses of TLP gene families have been carried out in many species, including plants and animals [7], with some TLP genes in plants known to be involved in defense against pathogens [40,41]. The TLP gene family has been comprehensively analyzed in A. thaliana, rice, grape, wheat, cotton, and melon [1,7,9-11]. In the current study, we identified the TLP genes in the G. barbadense genome, we analyzed the evolutionary relationships of GbTLPs, as well as the response to $V$. dahliae infection of a subset of GbTLP genes.

\subsection{Expansion of the GbTLP Gene Family in Sea Island Cotton}

In this study, we identified 90 putative TLP genes (GbTLP1 to GbTLP90) from the genome of sea island cotton. In a previous study, 91 TLP genes were identified mainly because different reference genomes and identification procedures including the selection of E-value were selected [11].

On the one hand, the results of gene duplication analysis suggested a mass of gene duplication events in the cotton genome. GbTLP genes were found to cluster into six tandem duplication event regions on six chromosomes (scaffolds) (Figure 2). Moreover, several tandem duplicated genes were classified into the same subfamily, implying that they may have originated from recent gene duplication events. In addition, the results of collinearity analysis indicated that segmental duplication was the main approach during duplication of the GbTLP gene family. These results clearly revealed that tandem duplication and segmental duplication were the major factors leading to the expansion of the TLP gene family in sea island cotton.

Natural selection analysis is widely used to estimate how natural pressures and evolutionary forces affect duplicated genes and the corresponding proteins encoded [41]. Selective pressure includes positive selection, purifying selection, and neutral selection. In sea island cotton, evolution of the majority of TLP genes was shown to be driven by purifying selection, whereas only three pairs of duplicated genes were brought about by positive selection, including the pairs GbTLP6 and GbTLP7, GbTLP18 and GbTLP57, and GbTLP30 and GbTLP72, indicating that six genes evolved by natural selection for mutations beneficial to the organism. A study on poplar TLP genes suggested that four TLP kinases and 10 TLPs underwent positive selection [2], indicating that the poplar TLPs may have experienced diversified natural selection processes, similar to that which occurred in the current study with the TLPs in sea island cotton. Most TLP sequences evolved under the influence of purifying (negative) selection pressure, whereas on only a few sites in $T L P$ sequences did positive selection operate during the evolutionary process, paralleling results from studies on the evolution of TLPs in A. thaliana, rice, and maize [42].

In addition, the causes of gene expansion may be due to different mechanisms of evolution. Liu et al. studied 118 TLP gene sequences, which were divided into nine groups, with the main genes belonging to the five groups IV, VI, VII, VIII, and IX, and they considered that the TLP genes originated about $1.0 \times 10^{9}$ years ago in the common ancestor of plants, animals, and fungi [2]. Compared with the TLPs in A. thaliana, which consist of 10 evolutionary groups, the TLPs in sea island cotton were also divided into 10 branches (Figure 3). It has been found that the TLPs of other species in evolutionary group 5 can respond to pathogenic and environmental stresses. For example, AT4G11650, also known as ATOSM34, encodes a protein which participates in the signaling pathway of $A$. thaliana to respond to pathogenic microorganisms and to salt stress [43].

\subsection{The Putative Regulatory Mechanisms of GbTLP Gene Expression}

A number of research studies have shown that TLPs are localized in the extracellular space, enhancing plant tolerance to various stresses [20,44]. TLP gene expression can be regulated by a variety of signals, such as SA, MeJA, abscisic acid, injury, UV, osmotic stress, and invasion by bacteria, fungi, viruses, and other organisms [45]. In the 
GbTLP promoter regions, we found a number of stress-responsive cis-elements, such as ARE, MBS, LTR, WUN-motif, and TC-rich repeats, which are responsive to biotic and abiotic stresses (Figure 5). We also found several phytohormone-associated regulatory elements in the GbTLP promoters, which indicated that the GbTLP gene family probably participates in phytohormone signaling pathways. Specifically, we noted the presence of ABRE, TGA/AuxRR-core, and GARE motif elements, which were associated with ABA, gibberellin, and auxin responses, respectively. According to reports, ABA accumulates under stress conditions, plays an important role in plant stress response and tolerance, and may coordinate reactive oxygen species (ROS) signaling pathways. For instance, tobacco TLPs induce yeast cell apoptosis through the accumulation of ROS, mediated by the RAS2/cAMP pathway [46], showing that the plant TLPs exhibit antimicrobial activity and may be related to the accumulation of ABA. Thus, multiple cis-regulated elements upstream of the gene coding sequence are essential for gene-specific expression. TLP gene expression is regulated by specific cis-elements in the promoter region. Plants respond to abiotic and biotic stresses in a variety of ways, including through the interaction between molecular and cellular changes to improve tolerance to abiotic stress and the control of related metabolic process mechanisms. These mechanisms involve multiple systems and are based on the synergistic effect of cascades of signal transduction networks, involving multiple genes, such as the response patterns between gene expression and hormones in plants. For example, gCC-boxes in the osmotic protein of tobacco gene promoter region are necessary for ethylene response [47]. However, evidence on the upstream regulation of GbTLPs and on the downstream factors regulated by GbTLPs at different levels is lacking. Furthermore, the relationship between the plant innate immune system and the mechanism regulating expression of GbTLP genes, as well as the non-coding miRNA-mediated stress tolerance in cotton, needs additional investigation.

\subsection{Expression Patterns of GbTLPs Responding to Verticillium Wilt Treatment}

In recent years, the antifungal activity of TLPs has been studied in detail. More than 20 TLPs from animals, fungi, and plants have been found to have antifungal activity. The TLPs in plants inhibited both pathogenic and nonpathogenic fungi by lysing fungal spores, as well as inhibiting spore germination and reducing the growth of young hyphae $[8,15,48]$. Most studies have shown that transgenic plants expressing recombinant TLPs delayed the development of a number of fungal diseases and enhanced the resistance of plants to pathogenic fungi $[9,12]$. The expression profiles of GbTLP genes in response to VW infection revealed their potential functions in response to challenge by pathogenic fungi and helped in understanding their biological roles. In this study, we found that the expression of 13 TLP genes including GbTLP30, GbTLP35, GbTLP39, GbTLP40, GbTLP47, GbTLP70, GbTLP71, GbTLP76, GbTLP77, GbTLP78, GbTLP779, GbTLP80, and GbTLP90, was significantly increased in VW-resistant cotton cv. Hai7124 following VW infection, suggesting that expression of these genes may correlate positively with cotton resistance to VW. These results were consistent with previous studies and supported the finding that many TLP genes exhibit antifungal activity [11]. For example, a recombinant CITLP27 protein isolated from watermelon drastically reduced the mycelial growth of several fungal phytopathogens [12]. In cherry tomato, recombinant protein LePR5 was found to exhibit antifungal activity against Cryptococcus laurentii [49]. However, some TLPs did not exhibit antifungal activity. Two osmotin-like proteins from latex lacked anti-fungal activity against several fungi phytopathogens [50]. Elderberry (Sambucus nigra) and barley TLP (HvPR5b, Pr22-1, and Pr22-2) were also found to exhibit no antifungal activity [51-54].

In the present work, three downregulated GbTLP genes, namely, GbTLP13, GbTLP49, and GbTLP72, were also identified in response to VW attack. The expressions of these genes were severely repressed, indicating that they may be involved in distinct physiological events rather than in host defense. Although numerous studies regarding the biological roles of TLPs have shown that proteins exhibited antifungal activities, detailed information on their functions is still uncertain when it comes to new species. More information about 
the TLPs reported here is needed to obtain a better understanding of the role played by these proteins in sea island cotton.

\section{Materials and Methods}

\subsection{Identification and Annotation of Sea Island Cotton TLP Genes}

The genomic database of G. barbadense (NAU) was downloaded from the Cotton Functional Genomics Database (CottonFGD) (https:/ / cottonfgd.org/) (10 March 2019). The HMM profile of the thaumatin domain (PF00314) was downloaded from the Pfam website (http:/ / pfam.xfam.org/) (25 March 2019) and was used as the query to identify all possible thaumatin-like sequences with HMMER software (http://hmmer.org) (25 March 2019). Furthermore, the conserved TLP domain of each candidate sequence was confirmed by Pfam. In the sea island cotton genome, we identified 90 TLP gene family members and obtained the details of individual TLP genes in sea island cotton, including genomic position, protein length, molecular weight $(\mathrm{kDa})$, isoelectric point, and exon-intron structure information through the CottonFGD (https:/ / cottonfgd.org/) (30 March 2019).

\subsection{Chromosomal Location Analysis}

The physical chromosome locations of all TLP genes were acquired from the genome sequence databases. According to the gene coordinates in the genome annotation, the chromosomal distribution of TLP genes in sea island cotton genome was drafted using Mapchart 2.3 software.

\subsection{Synteny and Collinearity Analysis}

We used BLAST (version 2.6.0) for the pairwise comparison of the filtered TLP sets of G. barbadense, and then MCscanX was employed to identify homologous regions, while syntenic blocks and duplicate gene classifications were evaluated using Circos-0.69. Default parameters were used in all the steps.

\subsection{Phylogenetic and Evolutionary Analysis}

We used two species to study the evolutionary relationship between the TLP genes, namely, sea island cotton and $A$. thaliana. The sequence data of $A$. thaliana were collected from the National Center for Biotechnology Information (NCBI) (http:/ / www.ncbi.nlm. nih.gov/protein/) (5 May 2019). Multiple alignments for all of the available and predicted TLP full-length protein sequences were performed using Clustal X2 with manual adjustment, where appropriate, for the alignment of the TLP domain. A phylogenetic tree was constructed using the neighbor-joining (NJ) method of MEGA 7.0.

\subsection{Gene Structure and Conserved Motif Analysis of the TLP Gene Family}

The genetic structure of GbTLP genes, including the exon-intron structure, was analyzed and mapped using the Gene Structure Display Server (GSDS 2.0). The conserved domain of TLP was analyzed using the online software MEME (http://meme-suite.org/) (15 July 2019), which was used to analyze the protein sequences of the G. barbadensedesignated GbTLPs. The maximum number of discovered modules was set to five, with the other parameters being tacit values.

\subsection{Prediction of Regulatory cis-Elements in the Promoter of GbTLPs}

The genomic sequences at $2.5 \mathrm{~kb}$ upstream of the transcription start site (TSS) of each GbTLP gene were extracted from the genome files of G. barbadense cv. Hai 7124 . The PlantCARE server (http:/ /bioinformatics.psb.ugent.be/webtools/plantcare/html/) (22 October 2019) was used to predict the transcriptional response elements of GbTLP gene promoters [55]. We obtained cotton miRNA sequences from the published literature $[56,57]$. GbTLP genes targeted by miRNAs were predicted by searching coding sequences (CDS) regions for sequences complementary to the cotton miRNAs using the psRNATarget server (http:/ / plantgrn.noble.org/psRNATarget/home) (18 November 2019) [58] with default 
parameters, except for maximum expectation $(E)=3.0$ and maximum unpaired energy $(\mathrm{UPE})=20.0$.

\subsection{Plant Materials and Treatments}

To study the responses of selected GbTLPs to challenge by $V$. dahliae in sea island cotton, a seed from G. barbadense cultivar cv. Hai 7124 was planted in potting soil at $25^{\circ} \mathrm{C}$ in a culture room with a $16 \mathrm{~h}$ light $/ 8 \mathrm{~h}$ dark cycle. Young plants at the four-true-leaf stage were inoculated with $V d 991$, a highly aggressive defoliating strain of $V$. dahlia, using the root dip inoculation method; the strain was cultured in complete medium at $25^{\circ} \mathrm{C}$ for 5 days, with the concentration of conidia for inoculation adjusted to $5 \times 10^{6}$ conidia $/ \mathrm{mL}$. The seedling leaves were harvested with three biological replicates at five time intervals $(0,6,12,24$, and $48 \mathrm{~h}$ ) after $V d 991$ inoculation. Each replicate consisted of five seedlings, which were immediately frozen in liquid nitrogen after harvest and stored at $-80^{\circ} \mathrm{C}$ until used for total RNA extraction. Total RNA from these samples was isolated using RNAprep Pure Plant Kit (polysaccharide- and polyphenolic-rich, DP441) (Tiangen, Beijing, China). The firststrand cDNA fragment was synthesized from total RNA using PrimeScript ${ }^{\circledR}$ RT Reagent Kit (Takara, Kusatsu, Japan). Then, the cDNA templates were subjected to 10-fold dilution and used for qPCR. The quality and concentrations of the isolated RNA samples were determined by $1 \%$ agarose gel electrophoresis and spectrophotometrically with a NanoDrop 2000 spectrophotometer (Thermo Fisher Scientific, Wilmington, DE, USA), respectively. Reverse transcription PCR was carried out using HiScript ${ }^{\circledR}$ II Q RT SuperMix for qPCR with gDNA wiper (R223) (Vazyme, Nanjing, China) on samples at the five timepoints.

\subsection{Quantitative Real-Time PCR Analysis}

Gene-specific primers used for qPCR amplification are listed in Table S2 and were designed using Primer Premier 5.0 by avoiding conserved regions within the members of the TLP gene family. Transcript levels were determined using a 7500 Real-Time PCR system (Applied Biosystems) and SYBR ${ }^{\circledR}$ Premix Ex Taq ${ }^{\mathrm{TM}}$ II (Tli RNaseH Plus) (Takara, Japan), with three technical replicates of each biological sample. The $20 \mu \mathrm{L}$ reaction volume contained $10 \mu \mathrm{L}$ of SYBR Premix Ex Taq II (Tli RNaseH Plus) $(2 \times), 0.8 \mu \mathrm{L}$ each of the PCR forward and reverse primers $(10 \mu \mathrm{M}), 0.4 \mu \mathrm{L}$ of ROX Reference Dye II $(50 \times), 2 \mu \mathrm{L}$ of cDNA, and $6 \mu \mathrm{L}$ of $\mathrm{ddH}_{2} \mathrm{O}$. The thermal cycling conditions were as follows: an initial denaturation step of $30 \mathrm{~s}$ at $95^{\circ} \mathrm{C}$, followed by 40 cycles of $5 \mathrm{~s}$ at $95^{\circ} \mathrm{C}$ for denaturation, $34 \mathrm{~s}$ at $60^{\circ} \mathrm{C}$ for annealing, and a melting curve step at $95^{\circ} \mathrm{C}$ for $15 \mathrm{~s}, 60^{\circ} \mathrm{C}$ for $1 \mathrm{~min}$, and $95^{\circ} \mathrm{C}$ for $15 \mathrm{~s}$. GbActin was used as an endogenous reference gene, and a melting curve analysis was performed. Samples at $0 \mathrm{~h}$ were used as calibrators in qPCR analyses. Cycle threshold $\left(C_{t}\right)$ values were used for the relative quantification of the input target number. The relative expression levels of genes were calculated using the $2^{-\Delta \Delta \mathrm{CT}}$ method, to normalize the variance among samples. For statistical analysis, standardization of gene expression data from three independent biological replicates per experiment was performed, and all reactions were performed with three technical replicates. The results were statistically analyzed using a Student's $t$-test at $p<0.05$.

\section{Conclusions}

We identified 90 putative GbTLP genes in the sea island cotton genome, which are distributed across 19 chromosomes and nine scaffolds and might be derived from polyploidization or segmental duplications. Analysis of the expression patterns of selected GbTLP genes in a VW-resistant cotton accession identified GbTLPs responsive to $V$. dahliae infection, implying their potential role in resistance to VW. Analysis of the promoter sequences revealed cis-acting elements associated with phytohormone signaling and stress response, with different members harboring distinct types and numbers, suggesting that individual members of the GbTLP gene family might be differentially regulated at the transcriptional level. Several GbTLPs contain target sites of miRNAs that have been previously shown to be associated with VW response, implying potential miRNA-mediated post- 
transcriptional regulation of GbTLPs in disease resistance. Collectively, our study provides a comprehensive analysis of the expression, regulation, and evolution of the GbTLP gene family, and it lays the foundation for further cloning and functional characterization of the GbTLP genes using a reverse genetics strategy.

Supplementary Materials: The following are available online at https:/ /www.mdpi.com/article/10 .3390/plants10122647/s1, Table S1. The TLP gene family in Gossypium barbadense; Table S2. Primer sequences used in the study.

Author Contributions: H.W., P.Z., and Y.Z. (Yunlei Zhao) conceptualized and designed the experiments; Y.Z. (Yilin Zhang) and W.C. performed the experiments and analyzed the data; Y.Z. (Yilin Zhang) wrote the paper; P.Z. and Y.Z. (Yunlei Zhao) revised the manuscript; X.S., H.G., and T.W. prepared the materials. All authors read and agreed to the published version of the manuscript.

Funding: This work was supported by the Natural Science Foundation of Henan Province of China (No. 202300410551) and the Central Public-interest Scientific Institution Basal Research Fund (No. 1610162021032).

Institutional Review Board Statement: Not applicable.

Informed Consent Statement: Not applicable.

Data Availability Statement: Not applicable.

Acknowledgments: We would like to thank Zhen Chen at Henan Agricultural University for support with the data analysis in this work.

Conflicts of Interest: The authors declare no conflict of interest.

$\begin{array}{ll}\text { Abbreviations } \\ \text { PR } & \text { Pathogenesis-related } \\ \text { TLP } & \text { Thaumatin-like protein } \\ \text { VD } & \text { Verticillium dahlia } \\ \text { pI } & \text { isoelectric point }\end{array}$

\section{References}

1. Zhao, J.P.; Su, X.H. Patterns of molecular evolution and predicted function in thaumatin-like proteins of Populus trichocarpa. Planta 2010, 232, 949-962. [CrossRef]

2. Liu, J.-J.; Sturrock, R.; Ekramoddoullah, A.K.M. The superfamily of thaumatin-like proteins: Its origin, evolution, and expression towards biological function. Plant Cell Rep. 2010, 29, 419-436. [CrossRef] [PubMed]

3. Hu, X.; Reddy, A. Cloning and expression of a PR5-like protein from Arabidopsis: Inhibition of fungal growth by bacterially expressed protein. Plant Mol. Biol. 1997, 34, 949-959. [CrossRef]

4. Ghosh, R.; Chakrabarti, C. Crystal structure analysis of NP24-I: A thaumatin-like protein. Planta 2008, 228, 883-890. [CrossRef]

5. Smole, U.; Bublin, M.; Radauer, C.; Ebner, C.; Breiteneder, H. Mal d 2, the thaumatin-like allergen from apple, is highly resistant to gastrointestinal digestion and thermal processing. Int. Arch. Allergy Immunol. 2008, 147, 289-298. [CrossRef]

6. Fierens, E.; Rombouts, S.; Gebruers, K.; Goesaert, H.; Brijs, K.; Beaugrand, J.; Volckaert, G.; Van Campenhout, S.; Proost, P.; Courtin, C.M.; et al. TLXI, a novel type of xylanase inhibitor from wheat (Triticum aestivum) belonging to the thaumatin family. Biochem. J. 2007, 403, 583-591. [CrossRef] [PubMed]

7. Shatters, R.G.; Boykin, L.; Lapointe, S.L.; Hunter, W.B.; Weathersbee, A.A. Phylogenetic and structural relationships of the PR5 gene family reveal an ancient multigene family conserved in plants and select animal taxa. J. Mol. Evol. 2006, 63, 12-29. [CrossRef] [PubMed]

8. Abad, L.R.; D’Urzo, M.P.; Liu, D.; Narasimhan, M.L.; Reuveni, M.; Zhu, J.K.; Niu, X.; Singh, N.K.; Hasegawa, P.M.; Bressan, R.A. Antifungal activity of tobacco osmotin has specificity and involves plasma membrane permeabilization. Plant Sci. 1996, 118, 11-23. [CrossRef]

9. Yan, X.; Qiao, H.; Zhang, X.; Guo, C.; Wang, M.; Wang, Y.; Wang, X. Analysis of the grape (Vitis vinifera L.) thaumatin-like protein (TLP) gene family and demonstration that TLP29 contributes to disease resistance. Sci. Rep. 2017, 7, 1-14. [CrossRef]

10. Liu, Y.; Cui, J.; Zhou, X.; Luan, Y.; Luan, F. Genome-wide identification, characterization and expression analysis of the TLP gene family in melon (Cucumis melo L.). Genomics 2020, 112, 2499-2509. [CrossRef] 
11. Li, Z.; Wang, X.; Cui, Y.; Qiao, K.; Zhu, L.; Fan, S.; Ma, Q. Comprehensive genome-wide analysis of thaumatin-like gene family in four cotton species and functional identification of GhTLP19 involved in regulating tolerance to verticillium dahlia and drought. Front. Plant Sci. 2020, 11, 575015. [CrossRef] [PubMed]

12. Chowdhury, S.; Basu, A.; Kundu, S. Overexpression of a new osmotin-like protein gene (SindOLP) confers tolerance against biotic and abiotic stresses in sesame. Front. Plant Sci. 2017, 8, 410. [CrossRef]

13. Monteiro, S.; Barakat, M.; Piçarra-Pereira, M.A.; Teixeira, A.R.; Ferreira, R.B. Osmotin and thaumatin from grape: A putative general defense mechanism against pathogenic fungi. Phytopathology 2003, 93, 1505-1512. [CrossRef]

14. Jayasankar, S.; Li, Z.; Gray, D.J. Constitutive expression of Vitis vinifera thaumatin-like protein after in vitro selection and its role in anthracnose resistance. Funct. Plant Biol. 2003, 30, 1105-1115. [CrossRef] [PubMed]

15. Rajam, M.V.; Chandola, N.; Goud, P.S.; Singh, D.; Kashyap, V.; Choudhary, M.L.; Sihachakr, D. Thaumatin gene confers resistance to fungal pathogens as well as tolerance to abiotic stresses in transgenic tobacco plants. Biol. Plant. 2007, 51, 135-141. [CrossRef]

16. Yasmin, N.; Saleem, M. Biochemical characterization of fruit-specific pathogenesis-related antifungal protein from basrai banana. Microbiol. Res. 2014, 169, 369-377. [CrossRef]

17. Wang, H.; $\mathrm{Ng}$, T.B. Isolation of an antifungal thaumatin-like protein from kiwi fruits. Phytochemistry 2002, 61, 1-6. [CrossRef]

18. Mahdavi, F.; Sariah, M.; Maziah, M. Expression of rice thaumatin-like protein gene in transgenic banana plants enhances resistance to fusarium wilt. Appl. Biochem. Biotechnol. 2011, 166, 1008-1019. [CrossRef]

19. Ojola, P.O.; Nyaboga, E.N.; Njiru, P.N.; Orinda, G. Overexpression of rice thaumatin-like protein (Ostlp) gene in transgenic cassava results in enhanced tolerance to Colletotrichum gloeosporioides f. sp. manihotis. J. Genet. Eng. Biotechnol. 2018, 16, 125-131. [CrossRef]

20. Singh, N.K.; Kumar, K.R.R.; Kumar, D.; Shukla, P.; Kirti, P.B. Characterization of a pathogen induced thaumatin-like protein gene AdTLP from arachis diogoi, a wild peanut. PLoS ONE 2013, 8, e83963. [CrossRef]

21. Acharya, K.; Pal, A.K.; Gulati, A.; Kumar, S.; Singh, A.K.; Ahuja, P.S. Overexpression of camellia sinensis thaumatin-like protein, CsTLP in potato confers enhanced resistance to macrophomina phaseolina and phytophthora infestans infection. Mol. Biotechnol. 2013, 54, 609-622. [CrossRef]

22. Breiteneder, H. Thaumatin-like proteins-A new family of pollen and fruit allergens. Allergy 2004, 59, 479-481. [CrossRef]

23. Munis, M.F.H.; Tu, L.; Deng, F.; Tan, J.; Xu, L.; Xu, S.; Long, L.; Zhang, X. A thaumatin-like protein gene involved in cotton fiber secondary cell wall development enhances resistance against Verticillium dahliae and other stresses in transgenic tobacco. Biochem. Biophys. Res. Commun. 2010, 393, 38-44. [CrossRef]

24. Zhang, T.; Jin, Y.; Zhao, J.-H.; Gao, F.; Zhou, B.; Fang, Y.-Y.; Guo, H.-S. Host-induced gene silencing of the target gene in fungal cells confers effective resistance to the cotton wilt disease pathogen Verticillium dahliae. Mol. Plant 2016, 9, 939-942. [CrossRef] [PubMed]

25. Xu, L.; Zhu, L.; Tu, L.; Liu, L.; Yuan, D.; Jin, L.; Long, L.; Zhang, X. Lignin metabolism has a central role in the resistance of cotton to the wilt fungus Verticillium dahliae as revealed by RNA-Seq-dependent transcriptional analysis and histochemistry. J. Exp. Bot. 2011, 62, 5607-5621. [CrossRef] [PubMed]

26. Zhao, Y.; Wang, H.; Chen, W.; Zhao, P.; Gong, H.; Sang, X.; Cui, Y. Regional association analysis-based fine mapping of three clustered QTL for verticillium wilt resistance in cotton (G. hirsutum L.). BMC Genomics 2017, 18, 661. [CrossRef] [PubMed]

27. Inderbitzin, P.; Subbarao, K. Verticillium systematics and evolution: How confusion impedes Verticillium wilt management and how to resolve it. Phytopathology 2014, 104, 564-574. [CrossRef]

28. Shaban, M.; Miao, Y.; Ullah, A.; Khan, A.Q.; Menghwar, H.; Khan, A.H.; Ahmed, M.M.; Tabassum, M.A.; Zhu, L. Physiological and molecular mechanism of defense in cotton against Verticillium dahliae. Plant Physiol. Biochem. 2018, 125, 193-204. [CrossRef] [PubMed]

29. Leone, P.; Menu-Bouaouiche, L.; Peumans, W.J.; Payan, F.; Barre, A.; Roussel, A.; Van Damme, E.; Rougé, P. Resolution of the structure of the allergenic and antifungal banana fruit thaumatin-like protein at 1.7-A. Biochimie 2006, 88, 45-52. [CrossRef]

30. Theis, T.; Stahl, U. Antifungal proteins: Targets, mechanisms and prospective applications. Cell. Mol. Life Sci. 2004, 61, 437-455. [CrossRef]

31. Ellegren, H. Comparative genomics and the study of evolution by natural selection. Mol. Ecol. 2008, 17, 4586-4596. [CrossRef] [PubMed]

32. Hurst, L.D. The Ka/Ks ratio: Diagnosing the form of sequence evolution. Trends Genet. 2002, 18, 486-487. [CrossRef]

33. Hu, W.; Hou, X.; Huang, C.; Yan, Y.; Tie, W.; Ding, Z.; Wei, Y.; Liu, J.; Miao, H.; Lu, Z.; et al. Genome-wide identification and expression analyses of aquaporin gene family during development and abiotic stress in banana. Int. J. Mol. Sci. 2015, 16, 19728-19751. [CrossRef]

34. Wittkopp, P.J.; Kalay, G. Cis-regulatory elements: Molecular mechanisms and evolutionary processes underlying divergence. Nat. Rev. Genet. 2011, 13, 59-69. [CrossRef]

35. Wightman, B.; Ha, I.; Ruvkun, G. Posttranscriptional regulation of the heterochronic gene lin-14 by lin-4 mediates temporal pattern formation in C. elegans. Cell 1993, 75, 855-862. [CrossRef]

36. Reinhart, B.J.; Slack, F.J.; Basson, M.; Pasquinelli, A.E.; Bettinger, J.C.; Rougvie, A.E.; Horvitz, H.R.; Ruvkun, G. The 21-nucleotide let-7 RNA regulates developmental timing in Caenorhabditis elegans. Nature 2000, 403, 901-906. [CrossRef] [PubMed]

37. Kurihara, Y.; Watanabe, Y. From the cover: Arabidopsis micro-RNA biogenesis through Dicer-like 1 protein functions. Proc. Natl. Acad. Sci. USA 2004, 101, 12753-12758. [CrossRef] 
38. Willmann, M.R.; Poethig, R.S. Conservation and evolution of miRNA regulatory programs in plant development. Curr. Opin. Plant Biol. 2007, 10, 503-511. [CrossRef] [PubMed]

39. Mallory, A.C.; Vaucheret, H. Functions of microRNAs and related small RNAs in plants. Nat. Genet. 2006, 38, S31-S36. [CrossRef]

40. Ho, V.S.; Wong, J.H.; Ng, T. A thaumatin-like antifungal protein from the emperor banana. Peptides 2007, 28, 760-766. [CrossRef]

41. Garcia-Casado, G.; Collada, C.; Allona, I.; Soto, A.; Casado, R.; Rodriguez-Cerezo, E.; Gomez, L.; Aragoncillo, C. Characterization of an apoplastic basic thaumatin-like protein from recalcitrant chestnut seeds. Physiol. Plant. 2000, 110, 172-180. [CrossRef]

42. Cao, J.; Lv, Y.; Hou, Z.; Li, X.; Ding, L. Expansion and evolution of thaumatin-like protein (TLP) gene family in six plants. Plant Growth Regul. 2016, 79, 299-307. [CrossRef]

43. Narasimhan, M.L.; Bressan, R.A.; D’Urzo, M.P.; Jenks, M.A.; Mengiste, T. Unexpected turns and twists in structure/function of PR-proteins that connect energy metabolism and immunity. Adv. Bot. Res. 2009, 51, 439-489.

44. Wang, Q.; Li, F.; Zhang, X.; Zhang, Y.; Hou, Y.; Zhang, S.; Wu, Z. Purification and characterization of a CkTLP protein from cynanchum komarovii seeds that confers antifungal activity. PLoS ONE 2011, 6, e16930. [CrossRef]

45. An, M.; Tong, Z.; Ding, C.; Wang, Z.; Sun, H.; Xia, Z.; Qi, M.; Wu, Y.; Liang, Y. Molecular characterization of the thaumatin-like protein PR-NP24 in tomato fruits. J. Agric. Food Chem. 2019, 67, 13001-13009. [CrossRef]

46. Narasimhan, M.L.; Damsz, B.; Coca, M.; Ibeas, J.; Yun, D.-J.; Pardo, J.M.; Hasegawa, P.M.; Bressan, R.A. A plant defense response effector induces microbial apoptosis. Mol. Cell 2001, 8, 921-930. [CrossRef]

47. Raghothama, K.G.; Maggio, A.; Narasimhan, M.L.; Kononowicz, A.K.; Wang, G.; D’Urzo, M.P.; Hasegawa, P.M.; Bressan, R.A. Tissue-specific activation of the osmotin gene by $\mathrm{ABA}, \mathrm{C}_{2} \mathrm{H}_{4}$ and $\mathrm{NaCl}$ involves the same promoter region. Plant Mol. Biol. 1997, 34, 393-402. [CrossRef]

48. Ramos, M.V.; de Oliveira, R.S.; Pereira, H.; Moreno, F.B.; Lobo, M.D.; Rebelo, L.M.; Brandão-Neto, J.; de Sousa, J.; Monteiro-Moreira, A.C.; Freitas, C.D.; et al. Crystal structure of an antifungal osmotin-like protein from Calotropis procera and its effects on Fusarium solani spores, as revealed by atomic force microscopy: Insights into the mechanism of action. Phytochemistry 2015, 119, 5-18. [CrossRef]

49. Guo, J.; Zhao, X.; Wang, H.; Yu, T.; Miao, Y.; Zheng, X. Expression of the LePR5 gene from cherry tomato fruit induced by Cryptococcus laurentii and the analysis of LePR5 protein antifungal activity. Postharvest Biol. Technol. 2015, 111, 337-344. [CrossRef]

50. Freitas, C.D.; Silva, M.Z.; Bruno-Moreno, F.; Monteiro-Moreira, A.C.; Moreira, R.A.; Ramos, M.V. New constitutive latex osmotin-like proteins lacking antifungal activity. Plant Physiol. Biochem. 2015, 96, 45-52. [CrossRef]

51. Singh, S.; Tripathi, R.K.; Lemaux, P.; Buchanan, B.B.; Singh, J. Redox-dependent interaction between thaumatin-like protein and $\beta$-glucan influences malting quality of barley. Proc. Natl. Acad. Sci. USA 2017, 114, 7725-7730. [CrossRef] [PubMed]

52. Osmond, R.I.W.; Hrmova, M.; Fontaine, F.; Imberty, A.; Fincher, G.B. Binding interactions between barley thaumatin-like proteins and (1,3)- $\beta$-D-glucans. JBIC J. Biol. Inorg. Chem. 2001, 268, 4190-4199. [CrossRef]

53. Zareie, R.; Melanson, D.L.; Murphy, P.J. Isolation of fungal cell wall degrading proteins from barley (Hordeum vulgare L.) leaves infected with Rhynchosporium secalis. Mol. Plant Microbe Interact. 2002, 15, 1031-1039. [CrossRef] [PubMed]

54. Van Damme, E.J.; Charels, D.; Menu-Bouaouiche, L.; Proost, P.; Barre, A.; Rougé, P.; Peumans, W.J. Biochemical, molecular and structural analysis of multiple thaumatin-like proteins from the elderberry tree (Sambucus nigra L.). Planta 2002, 214, 853-862. [CrossRef]

55. Lescot, M.; Déhais, P.; Thijs, G.; Marchal, K.; Moreau, Y.; Van de Peer, Y.; Rouzé, P.; Rombauts, S. PlantCARE, a database of plant cis-acting regulatory elements and a portal to tools for in silico analysis of promoter sequences. Nucleic Acids Res. 2002, 30, 325-327. [CrossRef]

56. Romanel, E.; Silva, T.F.; Corrêa, R.L.; Farinelli, L.; Hawkins, J.S.; Schrago, C.E.G.; Vaslin, M.F.S. Global alteration of microRNAs and transposon-derived small RNAs in cotton (Gossypium hirsutum) during Cotton leafroll dwarf polerovirus (CLRDV) infection. Plant Mol. Biol. 2012, 80, 443-460. [CrossRef]

57. Xie, F.; Sun, G.; Stiller, J.; Zhang, B. Genome-wide functional analysis of the cotton transcriptome by creating an integrated EST database. PLoS ONE 2011, 6, e26980. [CrossRef]

58. Dai, X.; Zhuang, Z.; Zhao, P.X. psRNATarget: A plant small RNA target analysis server (2017 release). Nucleic Acids Res. 2018, 46, W49-W54. [CrossRef] 